\title{
Three-Dimensional Analysis of Spontaneous and Thalamically Evoked Gamma Oscillations in Auditory Cortex
}

\author{
WILLIAM SUKOV AND DANIEL S. BARTH \\ Department of Psychology, University of Colorado, Boulder, Colorado 80309-0345
}

\begin{abstract}
Sukov, William and Daniel S. Barth. Three-dimensional analysis of spontaneous and thalamically evoked gamma oscillations in auditory cortex. J. Neurophysiol. 79: 2875-2884, 1998. The purpose of this study was to investigate interactions among laminar cell populations producing spontaneous and evoked high-frequency $(\sim 40 \mathrm{~Hz})$ gamma oscillations in auditory cortex. Electrocortical oscillations were recorded using a 64-channel epipial electrode array and a 16-channel linear laminar electrode array while electrical stimulation was delivered to the posterior intralaminar (PIL) nucleus. Spontaneous gamma oscillations, and those evoked by PIL stimulation, are confined to a location overlapping primary and secondary auditory cortex. Current source-density and principal components analysis of laminar recordings at this site indicate that the auditory evoked potential (AEP) complex is characterized by a stereotyped asynchronous activation of supra- and infragranular cell populations. Similar analysis of spontaneous and evoked gamma waves reveals a close spatiotemporal similarity to the laminar AEP, indicating rhythmic interactions between supra- and infragranular cell groups during these oscillatory phenomena. We conclude that neural circuit interactions producing the laminar AEP onset in auditory cortex are the same as those generating evoked and spontaneous gamma oscillations.
\end{abstract}

\section{INTRODUCTION}

Sensory information processing in the neocortex is associated with locally synchronized oscillations of membrane potential in the gamma frequency band $(\sim 40 \mathrm{~Hz})$ (Basar and Bullock 1992; Eckhorn et al. 1988; Engel et al. 1990; Gray and Singer 1989; Jones and Barth 1997; Prechtl 1994; Singer 1994). In the rodent auditory and somatosensory systems, gamma oscillations occur spontaneously (Barth and MacDonald 1996; Brett and Barth 1996, 1997; Franowicz and Barth 1995; Jones and Barth 1997; MacDonald and Barth 1995; MacDonald et al. 1996) but also may be evoked by appropriate sensory stimulation (Jones and Barth 1997). Gamma oscillations in auditory cortex persist after lesioning of the acoustic thalamus (Brett and Barth 1996) and other regions of the thalamic intralaminar nuclei (Barth and MacDonald 1996; Brett and Barth 1996) and basal forebrain (Brett and Barth 1996). This suggests that the cortex has the capacity to generate these phenomena in isolation through intrinsically oscillatory cells (Gray and McCormick 1996; Gutfreund et al. 1995; Llinás 1992; Llinás et al. 1991; Nuñez et al. 1992; Silva et al. 1991) and/or their network interconnections (Jefferys et al. 1996). Yet, recent evidence indicates that the thalamus plays a key role in the modulation of gamma oscillations in sensory cortex (Barth and MacDonald 1996; Brett and Barth 1997; MacDonald et al. 1997; Steriade and Amzica 1996; Steriade et al. 1996a,b). In the auditory system, activation of the specific projection nuclei through electrical stimulation of subdivisions of the medial geniculate nucleus (MG) inhibits spontaneous gamma oscillations (Barth and MacDonald 1996), whereas similar stimulation of the nonspecific posterior intralaminar nucleus (PIL) (Barth and MacDonald 1996; Brett and Barth 1997) and the auditory sector of the thalamic reticular nucleus (MacDonald et al. 1997) evokes intense and focal gamma oscillations in auditory cortex.

Our laboratory has developed methods for performing high spatial resolution multi-electrode mapping of gamma oscillations at the cortical surface. Using these techniques, the spatiotemporal distribution of oscillating activity may be determined and compared with the averaged evoked potential produced by transient auditory stimulation (AEP). This work has shown that spontaneous, sensory evoked, and thalamically evoked gamma oscillations share a common region of primary and secondary auditory cortex (Barth and MacDonald 1996; Brett and Barth 1996; Brett and Barth 1997; Franowicz and Barth 1995; Jones and Barth 1997; MacDonald and Barth 1995; MacDonald et al. 1996), suggesting that in all three conditions a similar population of cortical cells may be responsible for gamma neurogenesis. Furthermore, the gamma zone is a subset of auditory cortex activated during the AEP, suggesting that the transient AEP and gamma oscillations also may be produced by a common population of cells. In this experiment, we compared spatial distributions of the AEP to spontaneous and thalamically evoked gamma oscillations by mapping their surface potentials in auditory cortex. We then examined the intracortical neurogenesis of these phenomena using laminar microelectrode recording and mathematical modeling.

\section{METHODS}

\section{Surgical preparation}

Sprague-Dawley rats (300-500 g) were anesthetized for surgery with intramuscular injections of ketamine $(66 \mathrm{mg} / \mathrm{kg})$ and xylazine $(26 \mathrm{mg} / \mathrm{kg})$ and secured in a stereotaxic frame equipped with hollow ear bars. The eyes were coated with ophthalmic ointment, and normal body temperature was maintained with a regulated heating pad $\left(37.5^{\circ} \mathrm{C}\right)$. A unilateral craniotomy extending from bregma to lambda and lateral to the temporal bone exposed auditory cortex in the right hemisphere. The dura was reflected and pial surface frequently bathed with physiological saline.

\section{Stimulation}

Auditory click stimuli were presented using a high-frequency piezo-electric speaker placed $\sim 15 \mathrm{~cm}$ lateral to the contralateral 
ear. Clicks were generated by computer-controlled monophasic square-wave pulses $(0.3 \mathrm{~ms})$, shown in previous studies to activate most of auditory cortex in the rat. Subcortical electrical stimulation consisted of 500-ms trains of current pulses $(10-15 \mu \mathrm{A} ; 0.5-\mathrm{ms}$ duration; $500 \mathrm{~Hz}$ ) delivered with a stainless steel bipolar electrode positioned in the PIL ( $4.8 \mathrm{~mm}$ posterior to bregma, $3.0 \mathrm{~mm}$ lateral to midline, $6.4 \mathrm{~mm}$ ventral to the cortical surface).

\section{Recording}

Field potentials were mapped from the pial surface of primary and secondary auditory cortex with an $8 \times 8$ array of 64 silver electrodes (tip diameter $100 \mu \mathrm{m}$ ) covering a $3.5 \times 3.5 \mathrm{~mm}^{2}$ area in a single placement. After mapping evoked and spontaneous gamma power at the cortical surface, the epipial electrode array was removed and a laminar electrode array was inserted into the cortical region exhibiting maximum power (Fig. 3A). Laminar recordings were performed with a linear array of 16 stainless steel electrodes (tip diameter $75 \mu \mathrm{m}$; spacing $150 \mu \mathrm{m}$ ) extending to a depth of $2.25 \mathrm{~mm}$ from the cortical surface, fabricated according to the method of Jellema and Weijnen (1991). The top 10 electrodes recorded from the six layers of auditory cortex. Recordings were referred to a silver ball-electrode mounted in a burr-hole drilled in the left frontal bone and were simultaneously amplified and filtered (band-pass cutoff $=-6 \mathrm{~dB}$ at $1-100 \mathrm{~Hz}$, roll-off $=5 \mathrm{~dB} /$ octave ). The epipial array initially was positioned according to stereotaxic coordinates and then adjusted to similarly align the spatial distribution of AEP across animals. The characteristic spatiotemporal pattern of the AEP (Barth and Di 1990, 1991; Di and Barth 1992, 1993 ) also was used to identify the locations of primary (area 41) and secondary (areas 36 and 20) auditory cortex. Epipial and laminar AEPs were digitally sampled $(1,000 \mathrm{~Hz} ; 225 \mathrm{~ms})$ and averaged $(n=100)$. Ten to 20 single trials of epipial and laminar evoked and spontaneous gamma oscillations (randomized 5- to 15-s interstimulus interval ) were sampled digitally $(500 \mathrm{~Hz} ; 2 \mathrm{~s})$ and stored on disk for subsequent analysis.

\section{Epipial data analysis}

From each 2-s trial of evoked gamma oscillations, data from a baseline period $400 \mathrm{~ms}$ before stimulus onset and from a stimulus period consisting of the final $400 \mathrm{~ms}$ of PIL stimulation were analyzed. The baseline measurements were used to determine the location, spatial distribution, and amplitude of spontaneous gamma oscillations. Power spectral density estimates of the baseline and stimulation periods were computed at each electrode, with power in the gamma frequency band determined as the total power between 35 and $55 \mathrm{~Hz}$ (Fig. 2C, shaded region). Baseline and associated stimulus induced gamma power were subtracted to determine the spatiotemporal distribution and strength of gamma oscillations evoked by thalamic stimulation. Results for all stimulation trials were averaged for a given animal and a grand average was computed across all animals.

\section{Laminar data analysis}

To spatially resolve the laminar locations of extracellular current sinks and sources (transmembrane currents) along the axis of the linear electrode array, spatially smoothed current source-density (CSD) (Mitzdorf 1985; Nicholson and Freeman 1975; Rappelsberger et al. 1981; Vaknin et al. 1988) was computed according to the method of Vaknin et al. (1988), using the following equation (Rappelsberger et al. 1981)

$$
\frac{\mathrm{d}^{2} \phi}{\mathrm{d} z^{2}}=\frac{\begin{array}{c}
0.23 \phi(z-2 h)+0.08 \phi(z-h)-0.62 \phi(z) \\
+0.08 \phi(z+h)+0.23 \phi(z+2 h)
\end{array}}{h^{2}}
$$

This equation expresses CSD as the second spatial derivative of the smoothed values of potential $(\phi)$ measured in $150-\mu \mathrm{m}$ increments $(h)$ along an axis $(z)$ perpendicular to the cortical surface.

To characterize the spatiotemporal distribution of evoked and spontaneous gamma across the laminae, a method of phase-locked averaging was used (Barth and MacDonald 1996; MacDonald et al. 1996). The electrode at the cortical surface served as a temporal reference for averaging successive gamma waves in all 16 electrodes. Averaging was phase-locked to the CSD peaks (current sources) of gamma waves at this electrode site using an automated peak seeking algorithm. To limit noise, only gamma waves exceeding $1 \mathrm{SD}$ from the mean, computed across all single trials for a given animal, were included in the averaging procedure. This technique provides a single averaged cycle of CSD resulting from the gamma oscillating activity. Phase-locked averaging was used instead of a cross-correlation function because it provided a timeseries representation of laminar activity that could be compared directly with averaged results of the laminar AEP.

Finally, principal components analysis (PCA) was used to separate independent sources of covariance in the CSD across the laminar electrodes (Barth et al. 1988; Ruchkin et al. 1964). A matrix representing the averaged covariance in CSD between all pairs of laminar electrodes was first computed for a given animal and also averaged across animals. The eigenvectors of this matrix represented the axes, in electrode space, of the independent sources of covariation between electrodes (principal components), and the associated eigenvalues reflected the relative amount of the total variance accounted for by each principal component. A physiological interpretation of PCA in this application is that the principal components represent separate cellular populations that project distinct spatial patterns of CSD onto the laminar electrode array. The principal component loadings reflect these spatial patterns or how each identified cellular population makes a weighted contribution to the CSD recorded at each electrode. The principal component scores reflect the time course and polarity of CSD in each cellular population as it contributes to the recorded signal. Because the first two principal components accounted for $>90 \%$ of the variance of laminar AEPs, averaged evoked gamma waves, and averaged spontaneous gamma waves in all animals, only these components were retained for modeling and illustration.

\section{RES ULTS}

Figure $1 A$ shows the position of the $8 \times 8$ channel epipial electrode array in relation to primary (area 41 ; dark shaded region ) and secondary (areas 36 and 20; light shaded region) auditory cortex in the right hemisphere. The AEP (Fig. 1B) recorded from this position in five animals was similar to previous investigations (Barth and Di 1990, 1991; Brett et al. 1994; Di and Barth 1992, 1993) and quite consistent across animals (Fig. $1 B$; superimposed traces). The AEP was characterized by an early positive/negative biphasic response (labeled P1 and N1 in Fig. $1 C$, reflecting their polarity and sequence of occurrence) in area 41 (Fig. 1, B, frame $a$, and $C$, solid trace) that preceded a similar positive/negative biphasic response in area 36 (Fig. 1, B, frame $b$ and $C$, dashed trace) by $4-5 \mathrm{~ms}$. The P1/N1 wave was followed by a slow surface positive wave (P2; Fig. $1 C$ ). The highly stereotyped spatiotemporal characteristics of the AEP complex permitted consistent alignment of the electrode array across animals. Furthermore, the systematic latency shift between the rostrolateral and caudomedial region of the electrode array was typical of responses in areas 41 and 36, respectively, and permitted alignment of a template derived from previous histological studies of the areal distributions 


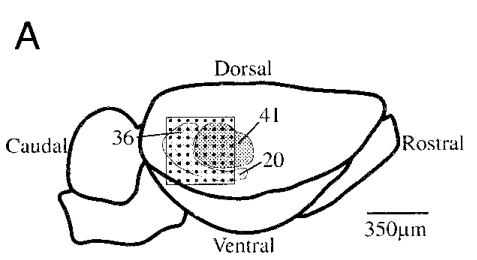

B

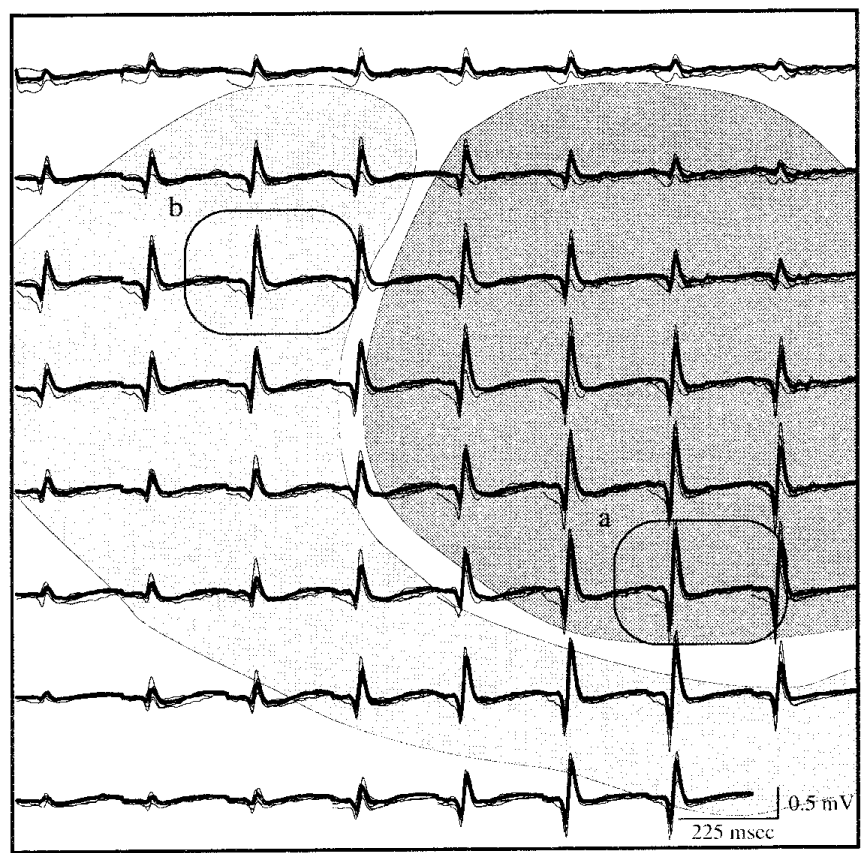

FIG. 1. Click-evoked epipial field potentials used to identify subregions of auditory cortex. A: epipial potentials were recorded using an $8 \times 8$ electrode covering a $3.5 \times 3.5 \mathrm{~mm}^{2}$ area of primary (area 41 ; dark shaded region) and secondary auditory cortex (areas 20 and 36; light shaded region) in the right hemisphere. $B$ : single animal averages (light traces) and the grand average (dark trace) of auditory evoked potentials (AEP) superimposed over the approximate locations of primary and secondary auditory cortex illustrate the characteristic epipial AEP pattern consisting of a sharp positive/negative biphasic response in area 41 (frame $a$ ) preceding a similar response in area 36 (frame $b$ ). $C$ : enlargement of the AEP complex recorded from area 41 (solid trace) depicts the initial biphasic positive/ negative response (P1 and N1, respectively) and the late positive (P2) component preceding the AEP complex in area 36 (dashed trace) by 4-5 ms.

of these regions (Barth and Di 1991; Krieg 1946; Patterson 1977; Zilles 1990) to the recording sites. The exact location and borders of areas 41, 36, and 20 indicated by this template must be considered approximate since no cytoarchitectural analyses of auditory cortex were performed in this study.

Electrical stimulation of the PIL evoked a characteristic cortical response consisting of a biphasic sharp wave, similar to the P1/N1 sequence of the AEP but with a disproportionately large negative component, followed by gamma oscillations sustained for the stimulus duration (Fig. 2, $A$ and $B$ ). Evoked gamma averaged across three animals, formed a focus straddling the border of primary and secondary auditory cortex (Fig. 2D), overlapping areas 41, 36, and 20, with little variability in the locations of maximum power for the individual animals (Fig. 2D, circles). Grand average gamma power during the baseline indicated that spontaneous
A
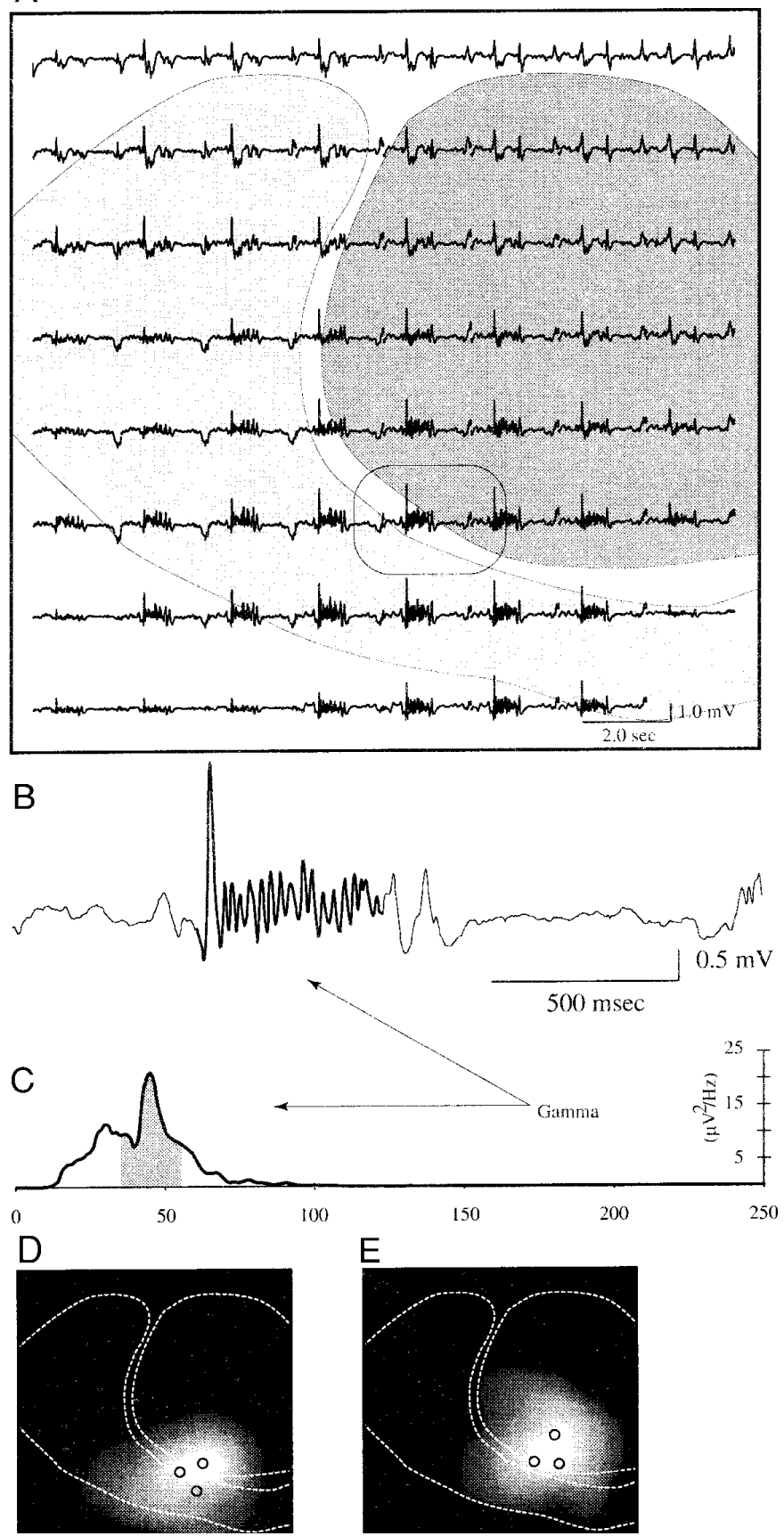

FIG. 2. Epipial oscillatory response in auditory cortex evoked by electrical stimulation of the posterior intralaminar nucleus of the thalamus (PIL). A: 500-ms stimulus train delivered to the PIL elicited epipial gamma oscillations in the caudolateral region of primary (dark shaded) and the lateral region of secondary (light shaded) auditory cortex. $B$ : characteristic evoked gamma response (enlarged from circled trace in $A$ ) was composed of a biphasic fast wave with a predominant N1 component, followed by oscillations persisting the stimulus duration (darkened region of the trace). $C$ : power spectral density (PSD) difference between baseline (400-ms prestimulus) and stimulation (period between 100- and 400-ms poststimulus-onset) peaked in the gamma band ( 35 and $55 \mathrm{~Hz}$, shaded region). Power of evoked gamma was summed within this bandwidth for subsequent mapping. $D$ : gray scale map of normalized gamma band power at all 64 electrodes, averaged across animals, indicates peak amplitude (white region) straddling the border between areas 41,36, and 20. Circles represent foci for averages of individual animals. $E$ : similar to $D$ but depicting gamma power for spontaneous bursts in the baseline alone. 

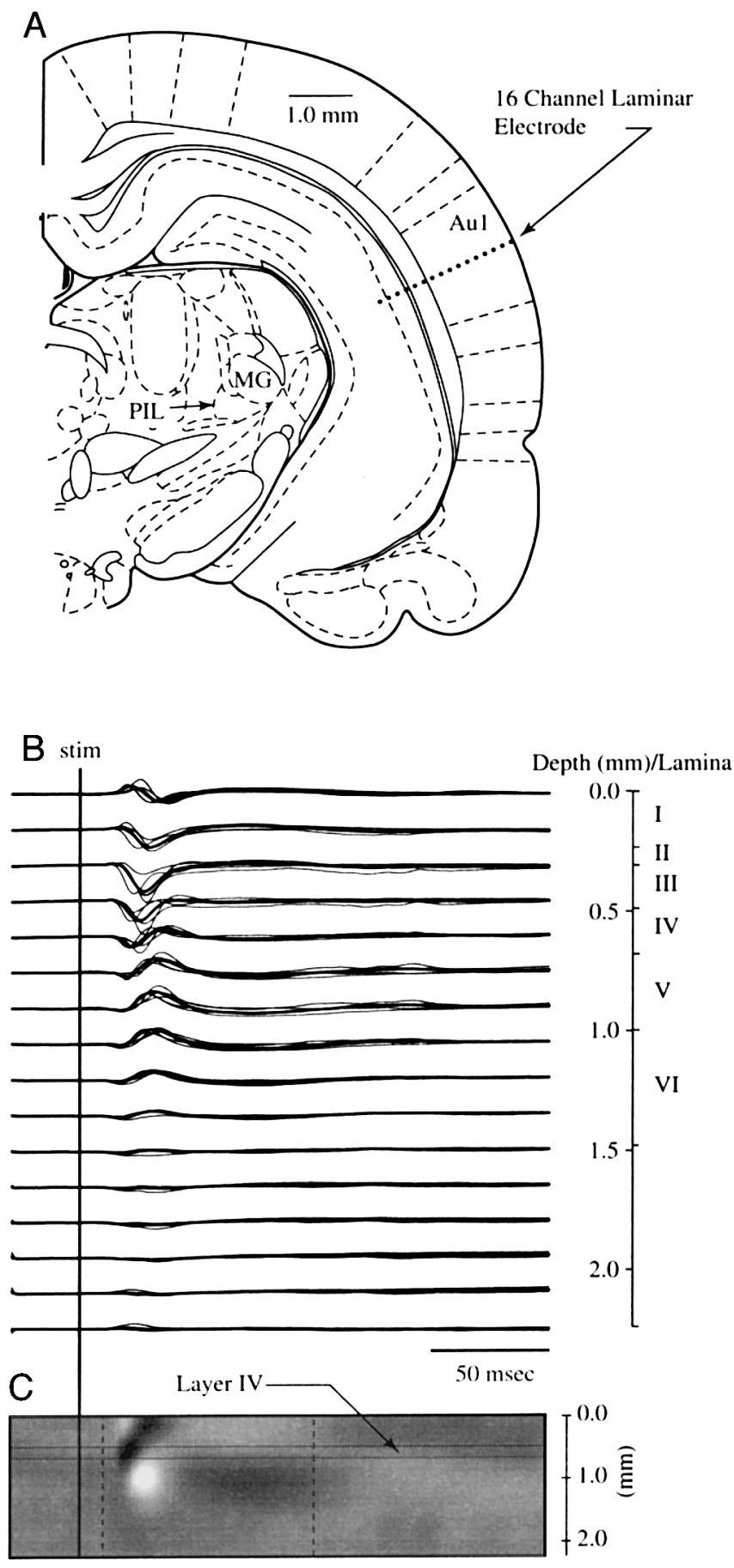

D

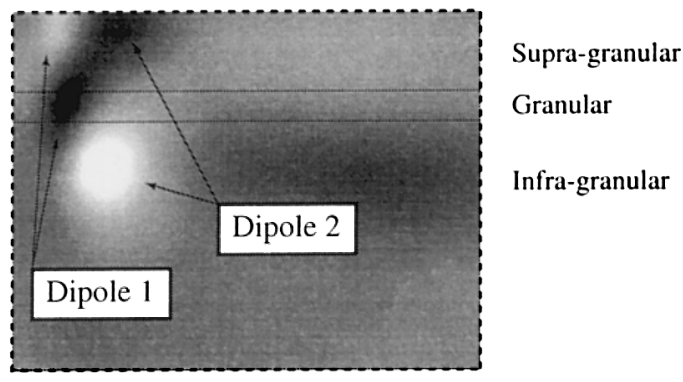

gamma bursts had a spatial distribution quite close to that of evoked gamma (Fig. $2 E$ ), with little variance across animals (Fig. 2E, circles).

Figure $3 B$ depicts laminar CSD of the AEP, averaged for four individual animals (light traces) and across animals (dark traces). The AEP in the most superficial electrode began with a biphasic fast wave produced by an extracellular current source (upward deflection) followed by a current sink (downward deflection) and corresponding in time to the P1/N1 of the surface potential. The fast wave reversed polarity in the depth and was constrained to the upper 10 electrodes of the array, indicating that the CSD pattern was produced locally within the laminae and not by volume conducted activity. However, the complex spatiotemporal pattern of polarity reversal throughout the laminae suggested that more than a single population of cells asynchronously contributed to the AEP. The AEP pattern, more easily visualized in the CSD maps of Fig. 3, $C$ and $D$, began with a source (white) in layer I with a complementary sink (black) in layers III-IV, characteristic of a vertically oriented equivalent current dipole produced by coherently summed postsynaptic currents in the apical dendrites of supragranular pyramidal cells (Fig. 3D; dipole 1 ). In contrast, the subsequent current sink at the cortical surface extended throughout the supragranular layers with a deeper complementary current source, indicating that a second current dipole (Fig. 3D; dipole 2) was produced by a population of infragranular pyramidal cells. Laminar recordings therefore indicated that the P1/N1 biphasic fast wave of the surface recorded AEP complex was produced by sequential activation of pyramidal cells in the supra- and infragranular layers, respectively. The AEP ended with a slow wave, corresponding in time to the surface recorded $\mathrm{P} 2$ wave, the CSD of which also indicated a vertically oriented current dipole but one that appeared to be composed of overlapping contributions of the supra- and infragranular populations.

Because activation of the supra- and infragranular layers during the AEP fast wave produced spatially and temporally

FIG. 3. Laminar distribution of the clicked-evoked AEP. A: multi-electrode consisting of 16 channels in a linear arrangement $(75-\mu \mathrm{m}$ diam surfaces; $150 \mu \mathrm{m}$ spacing) was used to measure field potentials to a depth of $2.25 \mathrm{~mm}$. On insertion into primary auditory cortex (Au1), the top 10 recording surfaces spanned all cortical layers. A scale representation of the multi-electrode, superimposed over a coronal map (adapted from Swanson 1992 ) corresponding to the anterior/posterior coordinates for electrode insertion, approximates the position of electrode surfaces during all recordings. Locations of the PIL and medial geniculate nucleus (MG) also are indicated. $B$ : current source-density analysis (CSD) identifies extracellular sources (upward deflection) and sinks (downward deflection). Laminar click-evoked AEP profiles for single animal (light traces) and grand (dark trace) averages suggest 2 asynchronously activated neuronal populations. First population is associated with a superficial source and a corresponding sink in layer IV. Beginning slightly before completion of activity by the 1st population, the 2 nd population exhibits a supragranular sink and infragranular source. A later slow wave composed of a supragranular source and infragranular sink concludes the evoked AEP. $C$ : gray scale map of the grand average CSD values more clearly depicts the spatiotemporal pattern of current sources (white) and sinks (black) during the AEP. Light horizontal lines bound layer IV. $D$ : enlargement of the area of $C$ encased in a dashed box illustrates 2 vertically oriented dipoles that are asynchronously active during the AEP, reflecting sequential activation of supragranular and infragranular pyramidal cells, respectively. Dipole 1 consists of a supragranular source with a corresponding sink in layer IV. Supragranular sink with a complementary infragranular source characterize dipole 2. 


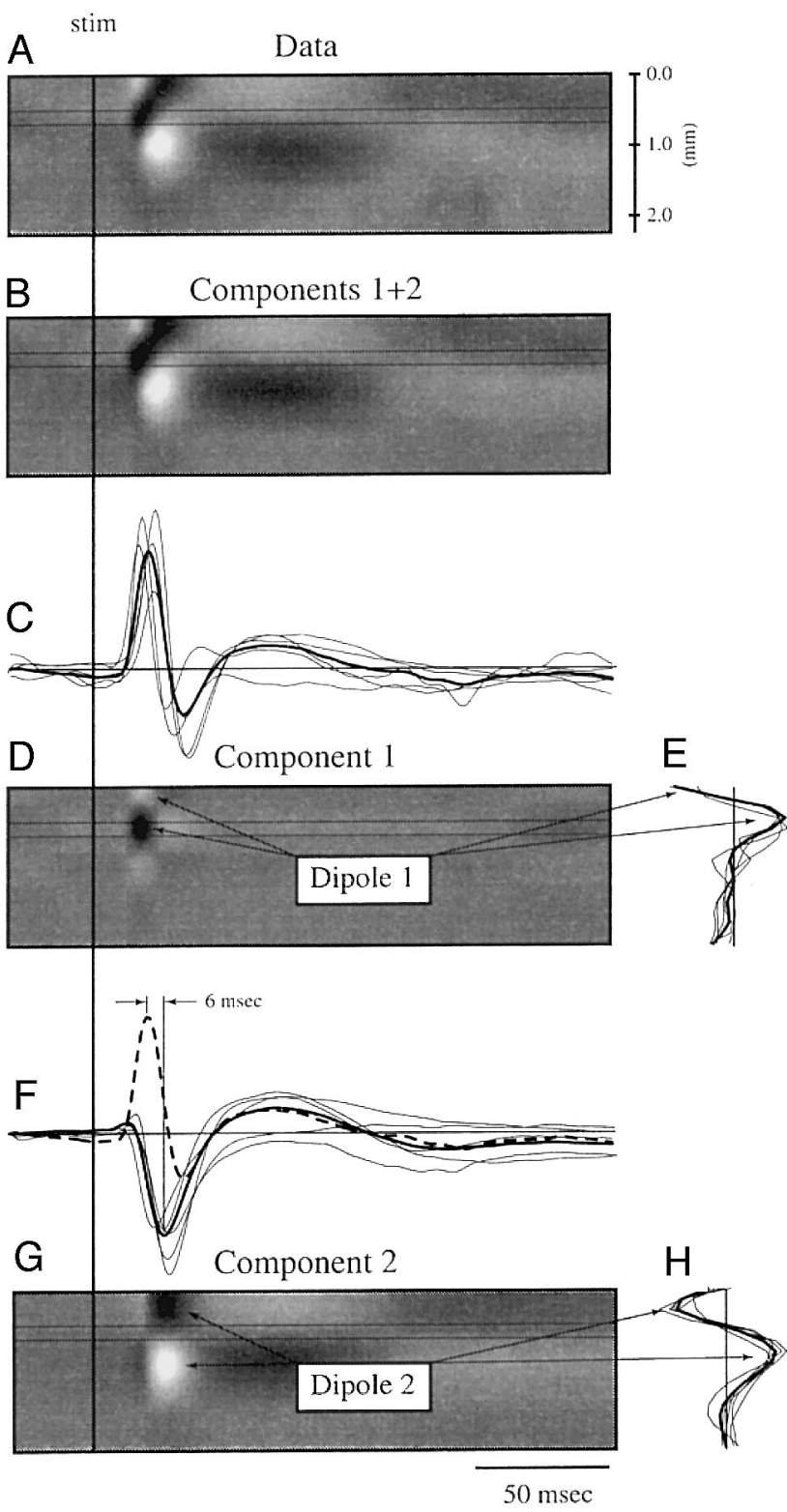

FIG. 4. Principal components analysis (PCA) of the laminar AEP. A: gray scale representation of the grand average AEP CSD values (from Fig. $3 C$ ). Dark vertical line indicates stimulus presentation in all plates. $B$ : model of the CSD profile in $A$, reconstructed from the 1 st 2 principal components, accounts for $>96 \%$ of the variance. $C-E$ : spatiotemporal pattern of component $1(D)$ is reconstructed by multiplying the temporal pattern of component scores ( $C$; dark trace) by the spatial pattern of component loadings ( $E$; dark trace). Scores and loadings for the individual animals are shown in superimposed light traces $(C$ and $E$ ). Component 1 reflects activity of an approximate current dipole confined to the supragranular layers (dipole 1). $F-H$ : similar to $C-E$ but depicting a reconstruction of component 2 , reflecting the independent activity of a current dipole in the infragranular layers (dipole 2). Grand average scores for component 1 (dashed trace) are superimposed on scores for component $2(F)$, illustrating a 6-ms delay between amplitude peaks of the supra- and infragranular dipoles. Composite model shown in $B$ consists of the sum of the separate maps for components 1 and 2 shown in $D$ and $G$, respectively.

distinct patterns in the laminar CSD profile, PCA was useful for modeling the independent contributions of each population to the total variance. Figure $4 A$ depicts the laminar CSD map of the AEP averaged across animals. Figure $4 B$ is a model of these data reconstructed using only the first two principal components and accounting for $96 \%$ of the variance. Two principal components similarly accounted 92 $98 \%$ of the variance in the individual animals. Maps reflecting the spatiotemporal contribution of the separate components (Fig. 4, $D$ and $G$ ) to the composite model (Fig. $4 B$ ) were computed by multiplying the loading patterns (Fig. $4, E$ and $H$ ) by their respective scores (Fig. 4, $C$ and $F$ ). The loading patterns reflect the weighted contributions of the components at each laminar electrode site, and the scores reflect the polarity and amplitude of the components over the time course of the AEP. Reconstructions of components 1 and 2 approximated separate activity of two distinct cell populations observed in the laminar AEP with spatial patterns similar to those expected from vertically oriented current dipoles in the supra- and infragranular layers (dipoles 1 and 2; Fig. 4, $D$ and $G$ ). Scores and loadings for the AEP averaged across animals (Fig. 4, $C, E, F$, and $H$; dark traces ), were quite similar to those computed for the individual animals (Fig. 4, $C, E, F$, and $H$; light traces). The amplitude peak in the supragranular layers (Fig. $4 F$; dashed trace) consistently preceded that of the infragranular layers (Fig. $4 F$; solid traces) by $\sim 6 \mathrm{~ms}$.

Averaged laminar CSD of gamma activity evoked by PIL stimulation also was constrained to the upper 10 electrode sites, confirming a cortical origin (Fig. $5 A$ ). This pattern appeared as a traveling wave with no clear point of polarity reversal in the depth (Figs. $5 B$ and $6 A$ ). However, PCA
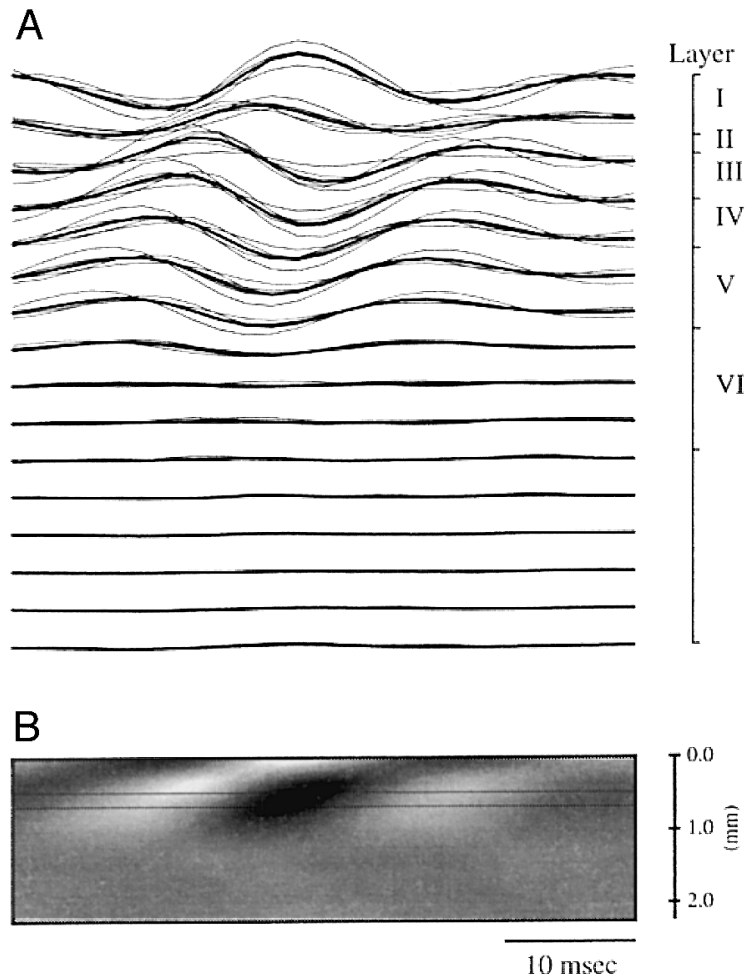

FIG. 5. Phase-locked averaging of CSD profiles was used to compare the spatiotemporal characteristics of gamma waves throughout the cortex with the surface electrode serving as the temporal reference. $A$ : gamma waves averaged for individual animals (light traces) and across animals (dark traces) demonstrate a latency shift through the laminae. $B$ : gray scale map of the grand averaged CSD depicts a traveling wave apparently sweeping from the depth toward the surface. 


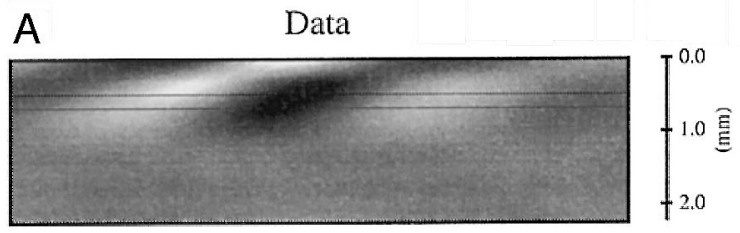

\section{B Components $1+2$}
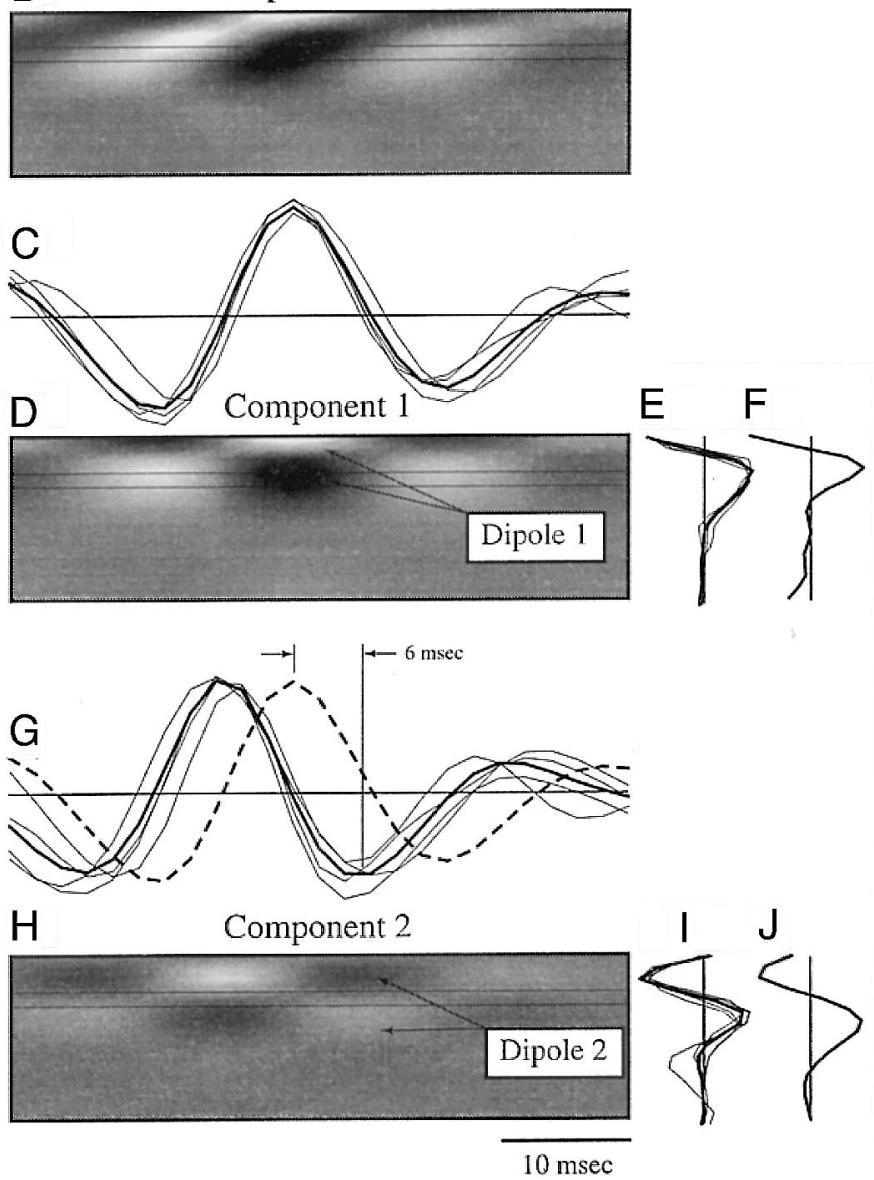

FIG. 6. Laminar PCA of evoked gamma. A: CSD profile of grand averaged gamma oscillations evoked by PIL stimulation as in Fig. 5B. B: reconstruction of this profile using the 1 st 2 principal components. $C-F$ : spatial and temporal reconstruction of the first principal component $(D)$, resulting from multiplication of component scores $(C)$ and loadings $(E)$, depicts a dipolar supragranular source and layer IV sink (dipole 1) bearing a close similarity to the P1 pattern characteristic of AEP component 1 (Fig. $4 D)$. In addition, nearly identical loadings for the AEP $(F)$ and evoked gamma $(E)$ indicate activation of similar neuronal populations during both phenomena. Scores and loadings were consistent for single animal (light traces) and grand (dark traces) averages. $G-J$ : reconstruction of component 2 demonstrated a dipole pattern (dipole 2), homologous to the N1 pattern of the AEP component 2 (Fig. $4 G$ ), characterized by a supragranular sink and infragranular source with dipole reversal in layer IV $(H)$. AEP and evoked gamma activities appear to recruit the same neuronal populations to produce dipole 2 as reflected by the similarity between AEP $(J)$ and evoked gamma $(I)$ loading patterns. Comparison of component 1 ( $G$, dashed trace) and 2 ( $G$, solid trace) scores indicate a 6-ms latency between the oscillations of the 2 neuronal populations.

modeling produced results that were similar to those obtained for the laminar AEP complex (Fig. 6). Modeling with two principal components accounted for $>95 \%$ of the CSD variance in gamma waves averaged across animals (Fig. 6, $B$ and $C, E, G$, and $I$; dark traces ) and for the individual animals (Fig. 6, $C, E$, $G$, and $I$; light traces ). Spatiotemporal reconstructions of the two components indicated that, similar to the P1/N1 wave of the AEP, evoked gamma waves were the result of asynchronous activation of supra- and infragranular pyramidal cell populations. The loadings (Fig. 6, $E$ and I) for both populations were dipolar and similar in laminar location and spatial distribution to those computed for the AEP (presented in Fig. $6, F$ and $J$ for comparison). The scores also indicated a 6-ms delay between oscillations in the supra- and infragranular populations (Fig. 6G).

The spatiotemporal pattern of laminar CSD during spontaneous gamma oscillations was nearly indistinguishable from that evoked by PIL stimulation. Again the averaged evoked gamma waves appeared as a traveling wave throughout the laminae (Fig. $7 A$ ), that could be separated into sequential activation of supra- and infragranular cell populations (Fig. $7, D$ and $G$, respectively) by PCA. Component scores (Fig.

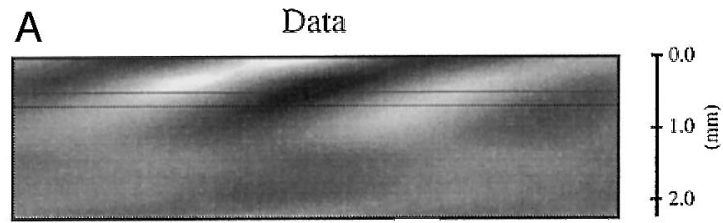

B Components $1+2$
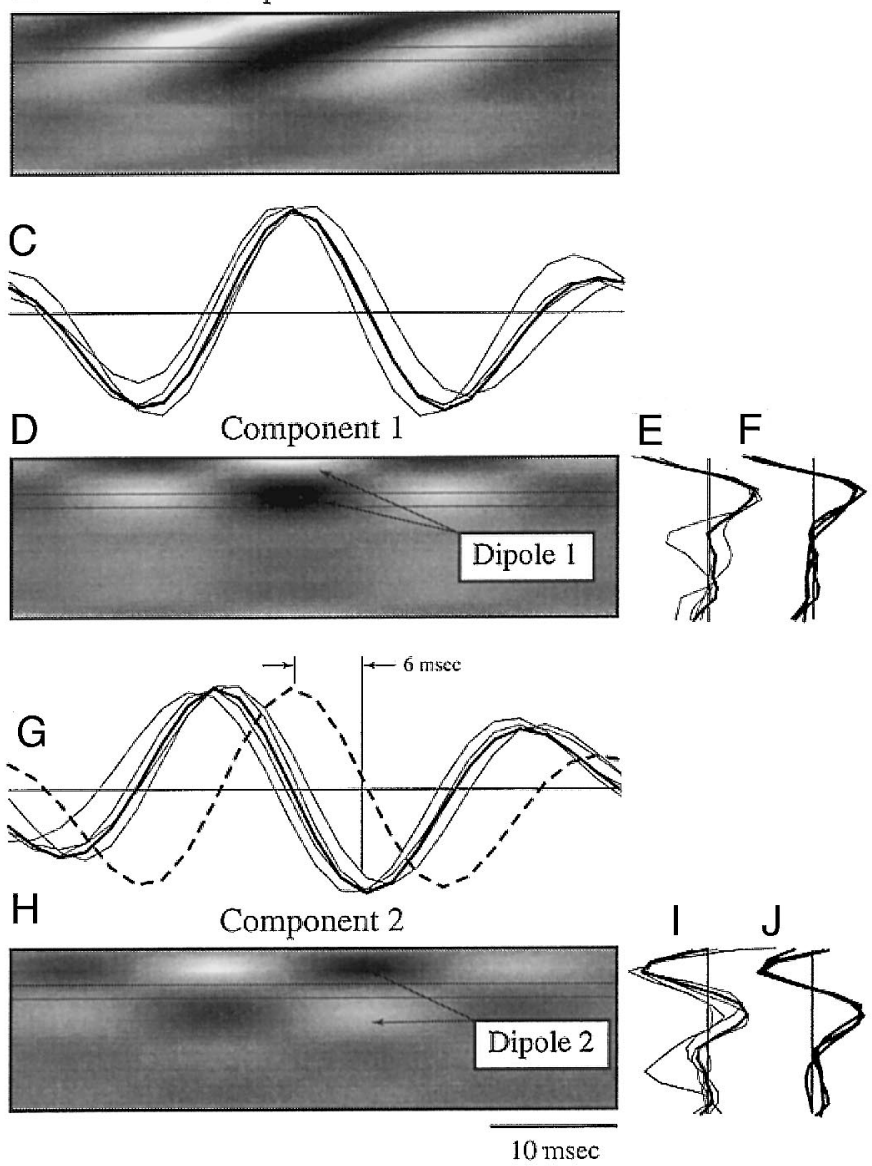

FIG. 7. Similar to Fig. 6 but depicting PCA results for averaged spontaneous laminar gamma CSD. Loading patterns for components 1 and 2 of the AEP and averaged evoked gamma are superimposed in $F$ and $J$, respectively, for comparison to loading patterns of the same components for averaged spontaneous gamma ( $E$ and $I)$. 
7, $C$ and $G$ ) indicated a similar 6-ms delay between oscillations in the two populations, and the component loadings (Fig. 7, $E$ and $I$ ) were similar to those obtained for both the averaged evoked gamma and the AEP complex (Fig. 7, $F$ and $J$ ).

\section{DIS C U S S ION}

Epipial recordings of the AEP, as well as spontaneous and thalamically evoked gamma oscillations in auditory cortex, replicate those obtained from previous studies. Click stimuli evoke a characteristic response at the cortical surface that is sufficiently stereotyped to consistently identify areas of primary and secondary auditory cortex (Barth and Di 1990). Spontaneous bursts of gamma oscillations occur in a subset of the region activated during the AEP, occupying an $\sim 2$ $\mathrm{mm}^{2}$ area that overlaps the caudal border between areas 41, 36, and 20 (Barth and MacDonald 1996; Brett and Barth 1996, 1997; Franowicz and Barth 1995; MacDonald and Barth 1995; MacDonald et al. 1996). When trains of electrical stimuli are applied to the PIL, the same subregion of auditory cortex produces large amplitude and continuous gamma oscillations during stimulation, usually continuing 100-200 ms poststimulus (Barth and MacDonald 1996; Brett and Barth 1997). Epipial recordings therefore suggest an association between populations of cells giving rise to the AEP complex and those responsible for generating both evoked and spontaneous gamma oscillations in auditory cortex, an association confirmed by the present laminar recording and analysis of potentials from the same region.

The physiological interpretation of laminar CSD analysis and PCA is based on several assumptions that have been reviewed in detail elsewhere (Barth et al. 1989; Mitzdorf 1985; Nicholson 1979; Nicholson and Freeman 1975; Ruchkin et al. 1964). Neural elements giving rise to the laminar CSD are assumed to resemble core conductors oriented perpendicular to the cortical surface. Based on their highly parallel geometry, vertical orientation, and discrete laminar organization of synaptic contact, the apical dendrites of cortical pyramidal cells are the most likely candidates contributing to the laminar CSD in sensory cortex (Barth et al. 1989; Mitzdorf 1985). Extracellular sinks are matched by complementary sources distributed along the apical dendrites throughout the laminae, and their laminar location and extent may be used to identify the location and extent of underlying pyramidal cell populations giving rise to the CSD. Onedimensional CSD computed perpendicular to the cortical laminae is only accurate if all extracellular current flow is oriented along the same axis and potentials along a plane parallel to the laminae are relatively uniform. Given that epipial recordings indicate the AEP and gamma activity subtend a region of $\sim 2 \mathrm{~mm}^{2}$ within which the laminar electrode subsequently was centered, the CSD computed here is necessarily an approximation of actual laminar transmembrane currents within this area.

The laminar synchrony of extracellular currents associated with activity of a given population of cells would be expected to produce a distinct pattern of covariance in the CSD within a subset of electrodes. These patterns of covariance justify the use of PCA to separate putative neuronal populations giving rise to the laminar CSD. Although the results of PCA are quantitatively valid, they must be regarded as only a model of underlying events; the order in which the principal components are extracted and their orthogonality is not based on physiological constraints (Wood and McCarthy 1984). Yet, these methods applied to the present data suggest a simple and physiologically interpretable activation pattern common to the AEP and gamma oscillations. The P1/N1 fast wave results from sequential activation of two approximately dipolar current sources in the supra- and infragranular laminae, respectively, that may be adequately modeled by two principal components. These results bear a close resemblance to earlier studies of laminar-evoked potentials in the auditory (Barth and Di 1990; Di and Barth 1993), somatosensory (Di et al. 1990), and visual (Mitzdorf 1985, 1987; Rappelsberger et al. 1981; Vaknin 1989) cortex, indicating a vertical cascade of excitatory activation at the evoked potential onset. The response begins with depolarization of proximal apical dendrites of supragranular pyramidal cells (P1), producing a sink in layer III and complementary source at the cortical surface ( surface positivity). This typeA activation pattern (Mitzdorf 1985) is due to thalamic afferents terminating directly, and indirectly through stellate cells of layer IV, on the supragranular pyramids. The N1 corresponds to a type-C activation pattern (Mitzdorf 1985) produced by depolarization of distal apical dendrites of infragranular pyramidal cells (surface negativity), mediated through ascending excitatory collateral fibers from the supragranular cells (Jones 1984). Similar models for the neurogenesis of the earliest evoked potential amplitude peaks have been proposed (Creutzfeldt and Houchin 1974; Goff et al. 1978; Landau 1967; Schlag 1973; Towe 1966), and analogous sequential and hierarchical models for the processing of thalamic input to the cortex also have emerged from single unit studies of sensory receptive field properties (Hubel and Wiesel 1968; Simons 1978).

Our finding that both evoked and spontaneous gamma oscillations are produced by similar laminar cell populations is in accord with recent laminar studies in cat, where it was observed that both spontaneous gamma oscillations, and those evoked by electrical stimulation of the pedunculopontine (PPT) nucleus, produced similar CSD patterns throughout the cortical laminae (Steriade and Amzica 1996; Steriade et al. 1996a). These investigators did not note the sequential activation of supra- and infragranular cell populations during gamma oscillations. However, in the first of the studies (Steriade et al. 1996a), cross-correlation functions were computed between extracellular potentials recorded with a surface electrode and an electrode penetrating various cortical depths. Because CSD was not computed, it is possible that very short latency shifts such as the 6-ms delay reported here may have been obscured by spatial smearing of overlapping volume conducted potentials from cells in distinct laminae. Interestingly, unit discharges consistently were associated with focal negative fast waves at depths of 0.15 and $0.7 \mathrm{~mm}$, and spike-triggered averages revealed an $\sim 2$ - to 3ms lag between surface and depth waves, similar to but shorter by several $\mathrm{ms}$ than the lag demonstrated in the present results. Furthermore, when CSD was computed in a subsequent report (Steriade and Amzica 1996), gamma oscillations again displayed a slight latency shift between the surface and depth electrode sites (see Fig. $1 B 3$ of this 
reference). This produced a diagonal pattern in the CSD topograms appearing as a wave of activation propagating between the cortical surface and depth similar to that depicted for averaged evoked and spontaneous gamma oscillations of the present study; a pattern that we have shown may be adequately modeled by the asynchronous activation of supra- and infragranular pyramids.

The present data indicate a latency shift of $6 \mathrm{~ms}$ between supra- and infragranular populations during gamma oscillations that is the same as that determined for the $\mathrm{P} 1 / \mathrm{N} 1$ of the AEP. The possibility that established intracortical neurocircuitry known to be responsible for the spatiotemporal dynamics of AEP onset may also participate in spontaneous and evoked gamma oscillations, provides further evidence that the cellular mechanism for gamma oscillations may be intracortical. This conclusion is consistent with findings in our laboratory demonstrating that spontaneous gamma oscillations in auditory cortex persist after destruction of the PIL (Barth and MacDonald 1996), as well as extensive lesioning of the acoustic thalamus eliminating all signs of the AEP (Brett and Barth 1996). Intracellular recordings recently have identified layer III-VI cortical cells capable of producing intrinsic oscillations in the gamma frequency band (Gray and McCormick 1996; Llinás 1992; Llinás et al. 1991; Nuñez et al. 1992; Silva et al. 1991). A unique population of cells in visual cortex produces gamma oscillations and accompanying bursts of action potentials when sufficiently depolarized either electrically or with appropriate sensory stimulation (Gray and McCormick 1996). These cells, of pyramidal morphology, are located consistently in layers II and III. A separate group of layer $\mathrm{V}$ pyramidal neurons also has been identified in rat sensorimotor cortex that displays an intrinsic rhythmicity (Silva et al. 1991). While the basal firing frequency of these layer $\mathrm{V}$ pyramids is typically below the gamma band, they fire at $35 \mathrm{~Hz}$ or more when sufficiently depolarized and, when appropriately coupled synaptically, could generate population rhythms in the gamma frequency range (Traub et al. 1989).

Intrinsically rhythmic layer III and V pyramidal cells may well be analogous to the supra- and infragranular populations identified in the present study. Histological examination indicates that the apical dendrites of cells within both of these populations extend into layer I (Gray and McCormick 1996; Silva et al. 1991), and synaptic activity therefore would be expected to produce laminar CSD patterns similar to those in Figs. 3-7. The layer III pyramids project excitatory axon collaterals to layers I and II (Gray and McCormick 1996) where the layer $\mathrm{V}$ pyramids display repeated branching of apical dendrites (Silva et al. 1991). Although no synaptic connection has been demonstrated yet between these two identified pyramidal populations, they provide a plausible neural circuit for sequential excitation of supra- and infragranular cells during gamma oscillations. Excitatory input from layer III to layer V pyramidal cells could establish a driven oscillator with a resonant frequency in the gamma band. This possibility is consistent with the average 6-ms time lag between gamma waves in the supra- and infragranular layers, resulting in a $90^{\circ}$ phase shift at $40 \mathrm{~Hz}$ that is optimal for resonance. However, given the nonlinear electrophysiology of dendrites (Koch et al. 1983), analogies to simple oscillators are only a first approximation. Further- more, the model ignores a likely additional role of interneurons, which also have been implicated in the synchronization of gamma oscillations in cortex (Llinás 1992; Llinás et al. 1991). The geometry of such interneuronal dendritic processes should produce self-cancelling extracellular potentials when summed over a population of synchronously active cells and subsequently would not be expected to produce detectable CSD patterns in laminar analysis performed here (Mitzdorf 1985).

Our data suggest that gamma oscillations in auditory cortex may reflect rhythmic interactions between supra- and infragranular layers, consisting of a repetition of the P1/N1 biphasic wave that characterizes the AEP onset. The question remains why electrical stimulation of the PIL is effective in evoking these oscillations, whereas stimulation of the MG fails to evoke gamma oscillations and will actually abort ongoing gamma activity (Barth and MacDonald 1996). Intracellular recordings demonstrate that fast oscillations emerge during depolarization of inherently oscillatory cortical cells (Gray and McCormick 1996; Llinás 1992; Llinás et al. 1991; Nuñez et al. 1992; Silva et al. 1991). Gamma oscillations in auditory (Franowicz and Barth 1995; MacDonald and Barth 1995), somatosensory (MacDonald and Barth 1995), and visual (Eckhorn et al. 1992) cortex are observed during the postinhibitory rebound phase of single trial transient evoked potentials. As proposed by Eckhorn and colleagues (1992), the late period of enhanced gamma oscillations probably results from enhanced cortical excitability. PIL stimulation therefore must provide predominantly excitatory input to the cortex in contrast to the archetypal excitatory inhibitory response sequence (Steriade 1984 ) characteristic of MG stimulation.

Although little is known about the function of the PIL, it is clearly part of the ascending auditory pathway. Anatomic tracing methods have demonstrated that the PIL receives its major afferent input from the inferior colliculus (Arnault and Roger 1987; Ledoux et al. 1987) and shares reciprocal connections with the ventral temporal cortex in the rat (Arnault and Roger 1987, 1990), in the same location as the evoked gamma focus. The midline and intralaminar nuclei of the thalamus historically have been characterized as nonspecific cortical projection systems (Jones 1985) that are parallel to the cortical projections from specific or relay nuclei of the thalamus, because they are known to receive afferents from many sources (Macchi and Bentivoglio 1986) and to have widespread cortical projection fields. Recent improvements in anatomic tracing techniques indicate that the rodent intralaminar nuclei may have greater specificity in their cortical projections than was previously assumed (Berendse and Groenewegen 1991) and may demonstrate a degree of modality specificity indicated by the present results.

Since the early anatomic studies of Lorente de Nó (1949), nonspecific thalamocortical projections have been regarded as modulatory. This conclusion has been based on anatomic evidence for their predominant termination in the supragranular cortical laminae (Herkenham 1980) and on functional evidence for their activating influence as measured in the electroencephalogram (Dempsey and Morison 1942). Whereas MG afferents terminate heavily in the middle cortical layers, those projecting from the intralaminar nuclei are 
anatomically distinct in that they also target layer I synaptic sites (Berendse and Groenewegen 1991; Cunningham and Levay 1986; Herkenham 1980; Jones 1975). The superficial termination of PIL fibers in layer I is probably responsible for the fact that the response evoked by PIL stimulation begins with a prominent negative wave at the surface as opposed to the positive/negative fast wave characteristic of MG stimulation. This surface negative wave reflects initial depolarization directly at distal apical dendrites without the typical cascade of activation from the middle cortical layers (Foster 1980; Olausson et al. 1989; Pellegrini et al. 1987; Rydenhag et al. 1986). In this way, PIL fibers may directly excite supra- and infragranular pyramidal cells without also activating slow inhibitory processes from interneurons in the middle cortical layers. Although inhibitory interneurons in layer IV are activated intracortically via axon collaterals from pyramidal cells, they also receive synaptic drive from the MG (Cipolloni and Keller 1989; White 1979, 1986). Recent evidence indicates that activation of GABAergic interneurons in layer IV, via MG afferents, produces an early inhibitory phase of evoked potentials in rat auditory cortex that corresponds in time to the $\mathrm{P} 2$ component of the surface recorded AEP. This inhibition results in rapid termination of early excitation (P1/N1) and suppression of late excitation (Metherate and Ashe 1995). Selectively exciting auditory cortex via PIL stimulation while bypassing MG projections to GABAergic interneurons in layer IV could result in repetitive P1/N1 fast waves (gamma oscillations) unchecked by inhibitory processes that interrupt the gamma oscillator.

This research was supported by Whitehall Foundation Grant S-97-06, a Sabbatical Fellowship and Grant in Aid to D. S. Barth from the Graduate School Council on Research and Creative Work at the University of Colorado at Boulder, and an Undergraduate Research Opportunities (UROP) Fellowship to W. Sukov at the University of Colorado at Boulder.

Address for reprint requests: D. S. Barth, Dept. of Psychology, University of Colorado, C.B. 345, Boulder, Colorado 80309.

Received 25 November 1997; accepted in final form 26 February 1998.

\section{REFERENCES}

Arnault, P. AND Roger, M. The connections of the peripeduncular area studied by retrograde and anterograde transport in the rat. J. Comp. Neurol. 258: 463-476, 1987.

ARnAUlt, P. AND Roger, M. Ventral temporal cortex in the rat: connections of secondary auditory areas Te2 and Te3. J. Comp. Neurol. 302: 110123, 1990.

BARTH, D. S. AND Di, S. Three dimensional analysis of auditory evoked potentials in rat neocortex. J. Neurophysiol. 64: 1527-1536, 1990.

BARTH, D. S. AND DI, S. The functional anatomy of auditory evoked potentials in rat neocortex. Brain Res. 565: 109-115, 1991.

Barth, D. S., Di, S., And Baumgartner, C. Principal component analysis of laminar currents produced by penicillin spikes in rat cortex. Soc. Neurosci. Abstr. 14: 6, 1988.

BARTh, D. S., Di, S., AND BAumgartner, C. Laminar cortical interactions during epileptic spikes studied with principal component analysis and physiological modeling. Brain Res. 484: 13-35, 1989.

BARTH, D. S. AND MacDonald, K. D. Thalamic modulation of high-frequency oscillating potentials in auditory cortex. Nature 383: 78-81, 1996.

BASAR, E. AND BuLlOCK, T. H. Induced rhythms in the brain. Brain Dynam. 4: 483,1992

BerENDSE, H. W. AND GROENEWEGEN, H. J. Restricted cortical termination fields of the midline and intralaminar thalamic nuclei in the rat. Neuroscience 42: 73-201, 1991.

BRETT, B. AND BARTH, D. S. The effects of subcortical lesions on evoked potentials and spontaneous high frequency (gamma-band) oscillating potentials in rat auditory cortex. Brain Res. 721: 155-166, 1996.
BRETT, B. AND BARTH, D. S. Subcortical modulation of high frequency (gamma-band) oscillating potentials in auditory cortex. J. Neurophysiol. 78: 573-581, 1997.

Brett, B., Di, S., WATKIns, L., AND BARTH, D. S. An HRP study of parallel thalamocortical projections responsible for the generation of mid-latency auditory evoked potentials. Brain Res. 647: 65-75, 1994.

Cipolloni, P. B. AND Keller, A. Thalamocortical synapses with identified neurons in monkey primary auditory cortex: a combined Golgi/EM and GABA/peptide immunocytochemistry study. Brain Res. 492: 347-355, 1989.

CreutzFeldt, O. D. AND Houchin, J. Neuronal basis of EEG-waves. In: Handbook of Electroencephalography and Clinical Neurophysiology, edited by A. Remond. Amsterdam: Elsevier, 1974, 2C, p. 5-55.

CunNingham, E.T.J. AND LEVAY, S. Laminar and synaptic organization of the projection from the thalamic nucleus centralis to primary visual cortex in the cat. J. Comp. Neurol. 254: 66-67, 1986

DEMPSEY, E. W. AND MORISON, R. S. The production of rhythmically recurrent cortical potentials after localized thalamic stimulation. Am. J. Physiol. 135: 293-300, 1942.

Di, S. AND BARTH, D. S. The functional anatomy of middle latency auditory evoked potentials: Thalamocortical connections. J. Neurophysiol. 68 425-431, 1992.

Di, S. AND BARTH, D. S. Binaural vs. monaural auditory evoked potentials in rat neocortex. Brain Res. 630: 303-314, 1993

Di, S., BAUMGARTNER, C., AND BARTH, D. S. Laminar analysis of extracellular field potentials in rat vibrissa/barrel cortex. J. Neurophysiol. 63: 832840, 1990.

Eckhorn, R., Bauer, R., Jordan, W., Brosch, W., Kruse, W., Munk, M., AND ReITBOECK, H. J. Coherent oscillations: a mechanism of feature linking in visual cortex? Biol. Cybern. 60: 121-130, 1988.

Eckhorn, R., Schanze, T., Brosch, M., Salem, W., and Bauer, R. Stimulus-specific synchronizations in cat visual cortex: multiple microelectrode and correlation studies from several cortical areas. In: Induced Rhythms in the Brain, edited by E. Basar and T. H. Bullock. Boston: QuinnWoodbine, 1992, vol. 4, p. 47-82.

Engel, A. K., KÖNIG, P., Gray, C. M., And Singer, W. Stimulus-dependent neuronal oscillations in cat visual cortex: inter-columnar interaction as determined by cross-correlation analysis. Eur. J. Neurosci. 2: 588-606, 1990

Foster, J. A. Intracortical origin of recruiting responses in the cat cortex. Electroencephalogr. Clin. Neurophysiol. 48: 639-653, 1980.

FRANOWICZ, M. N. AND BARTH, D. S. A comparison of evoked potentials and high frequency (gamma-band) oscillating potentials in rat auditory cortex. J. Neurophysiol. 74: 96-111, 1995.

Goff, W. R., Allison, T., And Vaughan, H. G., JR. The functional neuroanatomy of of event related potentials. In: Event-Related Brain Potentials in Man, edited by E. Callaway, P. Tueting, and S. H. Koslow. New York: Academic, 1978, p. 1-79.

GRAY, C. M. AND MCCORMICK, D. A. Chattering cells: superficial pyramidal neurons contributing to the generation of synchronous oscillations in the visual cortex. Science 274: 109-113, 1996.

Gray, C. M. AND Singer, W. Stimulus-specific neuronal oscillations in orientation columns of cat visual cortex. Proc. Natl. Acad. Sci. USA 86: $1698-1702,1989$.

Gutfreund, Y., Yarom, Y., AND Segev, I. Subthreshold oscillations and resonant frequency in guinea-pig cortical neurons: physiology and modeling. J. Physiol. (Lond.) 483: 621-640, 1995.

Herkenham, M. Laminar organization of the thalamic projections to the rat neocortex. Science 207: 532-535, 1980.

Hubel, D. H. AND Wiesel, T. N. Receptive fields and functional architecture of monkey striate cortex. J. Physiol. (Lond.) 195: 215-243, 1968.

Jefferys, J. G., Traub, R. D., And Whittington, M. A. Neuronal networks for induced " $40 \mathrm{~Hz}$ " rhythms. Trends Neurosci. 19: 202-208, 1996.

Jellema, T. AND WeiJnen, J.A.W.M. A slim needle-shaped multiwire microelectrode for intracerebral recording. J. Neurosci. Methods 40: $203-$ 209, 1991.

Jones, E. G. Possible determinants of the degree of retrograde neuronal labeling with horseradish peroxidase. Brain Res. 85: 249-253, 1975.

JONES, E. G. Identification and classification of intrinsic circuit elements in the neocortex. In: Dynamic Aspects of Neocortical Function, edited by G. M. Edelman, W. E. Gall, and W. M. Cowan. New York: Wiley, 1984, p. 6-40.

Jones, E. G. The Thalamus. New York: Plenum, 1985. 
Jones, M. S. AND BARTH, D. S. Sensory-evoked high frequency (gammaband) oscillating potentials in somatosensory cortex of the unanesthetized rat. Brain Res. 768: 167-176, 1997.

Koch, C., Poggio, T., And Torre, V. Nonlinear interactions in a dendritic tree: localization, timing, and role in information processing. Proc. Natl. Acad. Sci. USA 80: 2799-2802, 1983.

KRIEG, W.J.S. Connections of the cerebral cortex. I. The albino rat. Topography of the cortical areas. J. Comp. Neurol. 84: 221-275, 1946.

Landau, W. N. Evoked potentials. In: The Neurosciences, edited by G. C. Quarton, T. Melnechuk, and F. O. Schmitt. New York: Rockefeller Univ. Press, 1967, p. 469-482.

Ledoux, J. E., Ruggiero, D. A., Forest, R., Stornetta, R., And Reis, D. J. Topographic organization of convergent projections to the thalamus from the inferior colliculus and spinal cord in the rat. J. Comp. Neurol. 264: 123-146, 1987.

LlinÁs, R. R. Oscillations in CNS neurons: a possible role for cortical interneurons in the generation of $40-\mathrm{Hz}$ oscillations. In: Induced Rhythms in the Brain, edited by E. Basar and T. H. Bullock. Boston: QuinnWoodbine, 1992, vol. 4, p. 269-286.

LlinÁs, R. R., GRACE, A., AND YAROM, Y. In vitro neurons in mammalian cortical layer 4 exhibit intrinsic oscillatory activity in the $10-$ to $50-\mathrm{Hz}$ frequency range. Proc. Natl. Acad. Sci. USA 88: 897-901, 1991.

LORENTE DE Nó, R. Cerebral cortex: architecture, intracortical connections and motor projections. In: Physiology of the Nervous System, edited by J. F. Fulton. New York: Oxford Univ. Press, 1949, p. 274-313.

Macchi, G. And Bentivoglio, M. The thalamic interlaminar nuclei and the cerebral cortex. In: Cerebral Cortex, edited by G. Jones and A. Peters. New York: Plenum, 1986, p. 355-401.

MacDonald, K. D. AND BARTH, D. S. High frequency (gamma-band) oscillating potentials in rat somatosensory and auditory cortex. Brain Res. 694: 1-12, 1995.

MacDonald, K. D., Brett, B., AND Barth, D. S. Inter- and intra-hemispheric spatiotemporal organization of spontaneous electrocortical oscillations. J. Neurophysiol. 76: 423-437, 1996.

MacDonald, K. D., Fifkova, E., Jones, M. S., and Barth, D. S. Focal stimulation of the thalamic reticular nucleus induces focal gamma waves in cortex. J. Neurophysiol. 79: 474-477, 1997.

Metherate, R. AND Ashe, J. H. Synaptic interactions involving acetylcholine, glutamate, and GABA in rat auditory cortex. Exp. Brain Res. 107 59-72, 1995

MitZDORF, U. Current source-density method and application in cat cerebral cortex: investigation of evoked potentials and EEG phenomena. Physiol. Rev. 65: 37-100, 1985.

MitzDoRF, U. Properties of the evoked potential generators: current source density analysis of visually evoked potentials in cat cortex. Intern. J. Neurosci. 33: 33-59, 1987.

Nicholson, C. Generation and analysis of extracellular field potentials. In: Electrophysiological Techniques. Society for Neuroscience Short Course, 1979, p. 93-149.

Nicholson, C. And FreEman, J. A. Theory of current source-density analysis and determination of conductivity tensor for anuran cerebellum. $J$. Neurophysiol. 38: 356-368, 1975.

Nuñez, A., Amzica, F., and Steriade, M. Voltage-dependent fast (20$40 \mathrm{~Hz}$ ) oscillations in long-axoned neocortical neurons. Neuroscience 51: 7-10, 1992.

Olausson, B., Shyu, B. C., And Rydenhag, B. Projection from the thalamic intralaminar nuclei on the isocortex of the rat: a surface potential study. Exp. Brain Res. 75: 543-554, 1989.

Patterson, H. An Anterograde Degeneration and Retrograde Axonal Transport Study of the Cortical Projections of the Rat Medial Geniculate Body (PhD dissertation). Boston: Boston Univ., 1977.

Pellegrini, A., Currò-Dossi, R., Ermani, L., Zanotto, L., And Testa, $\mathrm{G}$. On the intracortical activity during recruiting responses: an analysis of laminar profiles before and after the topical application of GABA to the cortex. Exp. Brain Res. 66: 409-420, 1987.

PreChTL, J. C. Visual motion induces synchronous oscillations in turtle visual cortex. Proc. Natl. Acad. Sci. USA 91: 12467-12471, 1994.

Rappelsberger, P., Pockberger, H., And Petsche, H. Current source density analysis: methods and application to simultaneously recorded field potentials of the rabbit's visual cortex. Pflügers Arch. 389: 159170, 1981.

Ruchkin, D. S., Villegas, J., And John, E. R. An analysis of average evoked potentials making use of least mean square techniques. Ann. NY Acad. Sci. 115: 799-821, 1964.

Rydenhag, B., Olausson, B., Shyu, B. C., And Andersson, S. Localized responses in the midsuprasylvian gyrus of the cat following stimulation of the central lateral nucleus in thalamus. Exp. Brain Res. 62: 11-24, 1986.

SCHLAG, J. Generation of brain evoked potentials. In: Bioelectric Recording Techniques, edited by R. F. Thompson and M. M. Patterson. New York: Academic, 1973, p. 273-316.

Silva, L. R., Amitai, Y., AND Connors, B. W. Intrinsic oscillations of neocortex generated by layer 5 pyramidal neurons. Science 25: 432-435, 1991.

SIMONS, D. J. Response properties of vibrissa units in the rat SI somatosensory neocortex. J. Neurophysiol. 41: 798-820, 1978.

SINGER, W. Putative functions of temporal correlations in neocortical processing. In: Large-Scale Neuronal Theories of the Brain, edited by C. Koch and J. L. Davis. Cambridge, MA: MIT Press, 1994, p. 201-237.

STERIADE, M. The excitatory-inhibitory response sequence in thalamic and neocortical cells: state-related changes and regulatory systems. In: $D y-$ namic Aspects of Neocortical Function, edited by G. M. Edelman, W. E. Gall, and W. M. Cowan. New York: Wiley, 1984, p. 107-157.

STERIADE, M. AND AmZICA, F. Intracortical and corticothalamic coherency of fast spontaneous oscillations. Proc. Natl. Acad. Sci. USA 93: $2533-$ 2538, 1996.

Steriade, M., Amzica, F., and Contreras, D. Synchronization of fast $(30-40 \mathrm{~Hz})$ spontaneous cortical rhythms during brain activation. $J$. Neurosci. 16: 392-417, 1996a.

Steriade, M., Contreras, D., Amzica, F., And Timofeev, I. Synchronization of fast $(30-40 \mathrm{~Hz})$ spontaneous oscillations in intrathalamic and thalamocortical networks. J. Neurosci. 16: 2788-2808, 1996b.

Swanson, L. W. Brain Maps: Structure of the Rat Brain. Amsterdam: Elsevier, 1992.

Towe, A. L. On the nature of the primary evoked response. Exp. Neurol. 15: $113-139,1966$.

Traub, R. D., Miles, R., AND Wong, R. K. Model of the origin of rhythmic population oscillations in the hippocampal slice. Science 243: $1318-$ $1325,1989$.

VAKnIN, G. Major Excitatory Pathways in Rat Visual Cortex (PhD dissertation ). Kent, OH: Kent State Univ., 1989.

VAKnin, G., DiScenna, P. G., And Teyler, T. J. A method for calculating current source-density (CSD) analysis without resorting to recording sites outside the sampling volume. J. Neurosci. Methods 24: 131-135, 1988.

White, E. L. Thalamocortical synaptic relations: a review with emphasis on the projections of specific thalamic nuclei to the primary sensory areas of the neocortex. Brain Res. 180: 275-311, 1979.

White, E. L. Termination of thalamic afferents in the cerebral cortex. In: Cerebral Cortex, edited by E. G. Jones and A. Peters. New York: Plenum, 1986 , p. $271-285$.

Wood, C. C. And McCARThy, G. Principal component analysis of eventrelated potentials: Simulation studies demonstrate misallocation of variance across components. Electroencephalogr. Clin. Neurophysiol. 59: 249-260, 1984.

ZILLES, K. Anatomy of the neocortex: cytoarchitecture and myeloarchitecture. In: The Cerebral Cortex of the Rat, edited by B. Kolb and R. C. Tees. Cambridge: MIT Press, 1990, p. 77-112. 